\title{
AUTOMATIC ITERATIVE FITTING OF RUTHERFORD BACKSCATTERING SPECTRA FROM MULTIELEMENT SAMPLES
}

\author{
J.M. ERIDUN * and G.S. WAS ** \\ Department of Nuclear Engineering, University of Michigan, Ann Arbor, Michigan 48109, USA
}

A computer code (BASF) has been constructed to perform automatic iterative fitting of Rutherford backscattering spectra using only the experimental spectrum and the parameter set defining the experiment. The code may be used to analyze samples containing anywhere from two to five elements. The code output consists of the total amount of each element present and a composition versus depth profile.

The code's performance was verified on both computer generated and experimental backscattering spectra. Samples consisting of nickel substrates onto which layers of pure nickel and pure aluminum have been alternately evaporated in thicknesses of 130 and 100 $\AA$, respectively, were used to produce backscittering spectra. These spectra, when analyzed, demonstrated that the code was able to determine the total aluminum content to within $3 \%$ and the ratio of aluminum to nickel to within $1 \%$ of the thickness monitor readings taken during evaporation. The code has shown the ability to recognize sharp interfaces in well resolved spectra. The code performs equally well on slowly varying concentration profiles which are created during the annealing of layered samples. Limitations on the code and its use include the precise knowledge of the relevant experimental parameters used as input, and complete specification of all elements in the sample. The ultimate limits on the code's accuracy are the resolution of the spectrum and the accuracy of the computed stopping powers.

This code provides a significant advantage over other spectrum fitting codes in that the process is fully automated and does not require constant user interaction. Further, it provides the capahility of accurately determining concentration profiles in layered samples where the layer thickness is of the order $100 \AA$.

\section{Introduction}

Rutherford backscattering spectrometry (RBS) is a method for performing nondestructive surface chemical analysis using a charged particle accelerator and energy sensitive detectors. The technique relies on the kinematics of hard sphere scattering, which results in a different fractional energy transfer upon scattering that depends on the mass of the target atom. This makes it possible to analyze the elemental composition of a smooth surface to determine both the major alloying elements and impurity concentrations as a function of depth from the surface. Becasue of this capability, RBS is ideally suited to the analysis of surfaces consisting of multiple layers of two or more elements. Such surface structures frequently arise in ion beam mixing experiments where an inert gas ion beam is used to mix several layers of varying composition. In this study, we are concerned with producing a $600 \AA$ layer of approximately $40( \pm 10)$ volume percent $\gamma^{\prime}\left(\mathrm{Ni}_{3} \mathrm{Al}\right)$ in a $\gamma$ (nickel) matrix by ion beam mixing of multiple layers of nickel and aluminum. This two phase $\left(\gamma / \gamma^{\prime}\right)$ region has a width of approximately $10 \%$ in aluminum concentration, which means that a small $(1 \%)$ error in composi-

\footnotetext{
* Graduate student and research assistant.

** Associate professor.
}

tion will result in a large (10\%) error in the volume fraction of $\mathrm{Ni}_{3} \mathrm{Al}$. It is therefore necessary to be able to control and verify the surface composition to within $1 \%$.

While this may seem to be a straightforward task, there is no simple method of performing this analysis. Auger electron spectroscopy is not possible on such thin layers due to the high sputtering rates involved. Energy dispersive X-ray analysis requires the use of standards of approximately the same composition as the samples. Such standards are generally not available. Therefore, a method of accurately analyzing RBS spectra to determine composition profiles in thin layers needs to be developed.

Typically, this sort of analysis is performed by a computer code $[1,2]$ which models the backscattering process with the appropriate experimental parameters: geometry, beam energy, total current, and a guess of the probable elemental concentration profile. The modeling program then produces a spectrum which is compared to the experimental data, and the experimenter changes the input concentration profile to produce a better spectrum match. Alternatively, the spectrum can be fed to a mathematical deconvolution code which attempts to trace back from the experimental spectrum through the slowing down process and various spectrum broadening effects and solve a complicated set of equations for the concentration profile. Both methods suffer from 
the inherent lack of resolution caused by the finite detector resolution and energy straggling into and out of the sample. These effects make deconvolution a difficult, time consuming, imprecise procedure, and empirical fitting becomes a lengthy process of trying to adjust one elemental concentration to fit one portion of the spectrum, while compensating with another element to fit another section.

An automatic fitting routine (SQUEAKIE) has been written by Borgesen, et al. [3] which greatly simplifies the analysis of many spectra. This computer code determines the sample composition as a function of depth by solving a single set of linear equations for each element at each layer into the sample. The particular form of the equations and the solution procedure depend on both how much is known beforehand about the sample being analyzed and the form of the measured spectrum. The set of equations derived for each layer is generally over-determined, and some simplification such as ignoring the signal from a light element, or measuring the absolute "Q-value" - is necessary to create a solvable system. The target components are assumed to differ sufficiently so as to yield individually separable signals, except perhaps for one of the elements. With these restrictions, the code works very well in analyzing spectra from multicomponent samples.

In the present work, a similar analysis routine has been devised. The present code, BASF, is a modification of a manual fitting routine, RSVP [1], which has been revised to perform automatic iterative fitting of RBS spectra using only the experimental spectrum and the parameters defining the experiment as input. This new program differs from SQUEAKIE in many ways. Most importantly, as an iterative method, it allows the fitting of multiple element spectra with multiple overlapping signals by subtracting an estimated background signal at each step. On successive iterations, the background estimate improves, leading to better concentration calculations, which leads to better background estimates, etc. In effect, the code performs a trial backscattering experiment on each iteration and adjusts the sample composition until the simulated spectrum matches the measured spectrum. This method is applicable to all spectra, assuming the requircd cxperimental input parameters (detection geometry, energy calibration, and elements present in the sample) are known. Second, as a revision of a fitting program, the mathematical approach is less abstract than that found in Borgesen's paper, leading to a more intuitive derivation of the fitting equations. This approach also allows for more detail to be included in the spectrum simulation. For example, the signal from each element in each layer is treated differently with respect to the energy straggling it encounters, resulting in a more precise computed spectrum. Finally, the present code is less general than SQUEAKIE in that it will treat only backscattering experiments, whereas SQUEAKIE can be used to analyze spectra resulting from experiments involving nuclear reactions as well.

This new code, BASF, was tested first against computer generated spectra and then on actual experimental spectra obtained from multicomponent samples prepared by vacuum evaporation and analyzed using the 2 MV tandem ion accelerator facility in the Materials Science and Technology Division at Argonne National Laboratory. A description of the code, its capabilities and test results are presented in the following sections.

\section{Description}

The code (BASF - backscattering spectrum fitter) creates a spectrum by modeling the important processes which contribute to the detector signal. These are the slowing down and straggling of the beam particles as they travel into the sample, the backscattering event itself, the slowing down and straggling of the particles on the way out of the sample, and the actual detection event. The slowing down of the particles is accomplished by computing stopping powers using a tabulated six parameter quintic fit to experimental and extrapolated data [4]. The stopping power in multielement regions is assumed to be that obtained by weighting the individual stopping powers with the element's concentration (Bragg's rule). This assumption has been tested with helium stopping and found to be accurate to within a few percent [5]. The Rutherford cross section is used to compute the probability of a backscattering event:

$$
\begin{aligned}
\frac{\mathrm{d} \sigma}{\mathrm{d} \Omega}= & \left(\frac{z_{1} z_{2} e^{2}}{4 E}\right)^{2} \\
& \times \frac{4}{\sin ^{4} \theta} \frac{\left\{\left[1-\left(\left(M_{1} / M_{2}\right) \sin \theta\right)^{2}\right]^{1 / 2}+\cos \theta\right\}^{2}}{\left[1-\left(\left(M_{1} / M_{2}\right) \sin \theta\right)^{2}\right]^{1 / 2}},
\end{aligned}
$$

where

$\frac{\mathrm{d} \sigma}{\mathrm{d} \Omega}$ - scattering cross section per unit solid detection angle,

$z_{1}=$ atomic number of projectile,

$z_{2}=$ atomic number of target,

$M_{1}=$ mass of projectile,

$M_{2}=$ mass of target,

e = electronic charge,

$E \quad=$ projectile energy,

$\theta=$ backscattering angle,

and the subscripts 1 and 2 refer to projectile and target, respectively.

The energy straggling of the beam is modeled with the Bohr formula, wherein the variance of the Gaussian 
describing the encrgy distribution of the beam is assumed to increase by an amount:

$\Omega_{\mathrm{B}}^{2}=4 \pi\left(z_{1} e^{2}\right)^{2} N Z_{2} t$.

\section{where}

$N=$ atomic density of target,

$t=$ layer thickness,

$\Omega^{2}=$ straggling energy variance,

and the remaining symbols are as defined earlier. This relation applics to an ion traversing a layer of material 2 of thickness $t$ and atom density $N$. Straggling results in loss of resolution in the final spectrum. Another source of resolution loss is the detector itself, which typically has an energy resolution of about $15 \mathrm{kV}$ fwhm. All these sources of spectrum broadening are lumped into a single value of variance for computational purposes. and this value is computed for the signal from each element at each depth location in the sample, so that all signals suffer their allotted degree of spreading. This final value is computed as:

$\Omega_{i j}^{2}=K_{i}^{2} \Omega_{\mathrm{B}}^{2}(\mathrm{IN})+\Omega_{\mathrm{B}}^{2}(\mathrm{OUT})+\alpha(\mathrm{fwhm})^{2}$,

where

$\Omega_{u}=$ total variance of signal originating from element $i$ in layer $j$,

$K_{i} \quad=$ kinematic factor for scattering from element $i$, $\Omega_{\mathrm{B}}^{2}(\mathrm{IN})=$ Bohr straggling through material between surface and layer $j$ on the inward path,

$\Omega_{\mathrm{B}}^{2}($ OUT $)=$ Bohr straggling through material between layer $j$ and surface on the outward path,

fwhm $=$ full width at half maximum of detector resolution,

$\alpha \quad=8 \ln 2$, the factor necessary to convert from fwhm to Gaussian variance.

The code uses the initial concentration profile provided by the user to divide the sample into layers of constant stopping power. The composition and atom densities of these layers may change, but the restriction that the stopping power remain constant is a natural constraint which provides stability to the calculations. In this way, the magnitude of the signal from any element in a given layer will vary, but not the region of the spectrum to which the signal contributes. The signals produced by each element are stored in large, necessarily sparse response matrices of dimension (number of spectrum channels) $\times$ (number of layers). A spectrum is produced by performing a matrix multiplication of each response matrix with the vector giving the concentration profile of the associated element and summing the resultant partial spectra. A pointer matrix is used to keep track of which positions in each response matrix are filled, thereby avoiding needless multiplication operations. This ability to attribute a portion of the signal in any given region of the spectrum to different elements in different layers is what makes the iterative fitting of real spectra possible.

\subsection{Two element solution procedure}

The number of counts in a particular detector channel due to events occurring in a given layer is proportional to the following quantities: the integrated beam current striking the sample, the solid angle covered by the detector, the channel width, the scattering cross section for particles at the appropriate energy, the concentration of the particular element scattering into that channel $\left(N_{i}\right)$, and the inverse of the total stopping power of all elements in that layer $\left(\epsilon_{i}\right)$. Since stopping power is proportional to concentration, these last two variables are related. This relationship makes it possible to solve directly for the composition profile in a sample which is known to contain only two elements. This is done by noting that the signal in the leading edge of the spectrum comes from the heaviest element in the first layer of the sample. The thickness of this first layer (in total stopping power) determines the height of the signal in the leading edge, and so the ratio of element two (the light element) to element one (the heavy element) can be adjusted to obtain the proper signal height, as follows,

$$
\begin{aligned}
\text { signal height } & =N_{\mathrm{I}} /\left(N_{\mathrm{I}} \epsilon_{1}+N_{2} \epsilon_{2}\right) \\
& =1 /\left(\epsilon_{1}+\left(N_{2} / N_{1}\right) \epsilon_{2}\right) .
\end{aligned}
$$

Some small error $(\simeq 10 \%)$ will be introduced by the fact that the shapes of the stopping power curves for the two elements are somewhat different. This is easily resolved, however, by simply repeating the calculation once. As one moves further into the sample, a region will be found in which the light element signal from the first layer overlaps the heavy element signal from the deeper layer, fig. 1. Since the light element concentration is already known, this component can be computed and subtracted out, leaving the same calculation for con-

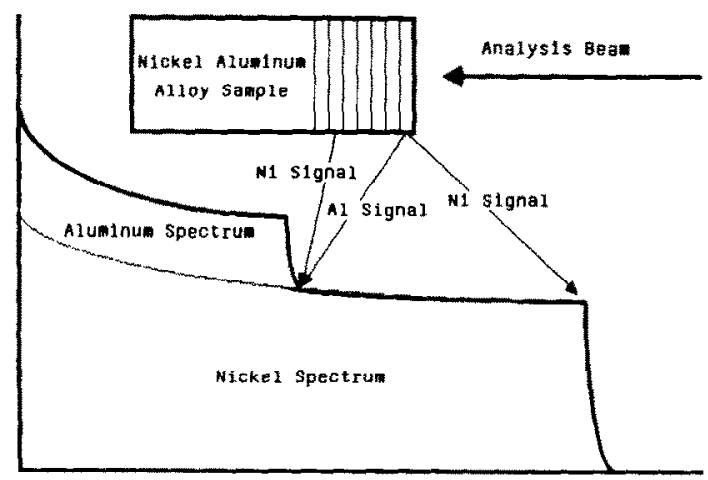

Fig. 1. Signals from different elements in different layers of a homogeneous alloy sample, showing signal overlap. 
centrations in the deep layers as was performed in the first.

\subsection{Three or more element solution procedure}

In a three element sample, this procedure cannot be followed due to the lack of an independent condition necessary to specify the balance between the stopping powers provided by the two lighter components. To resolve this situation, the concentrations of the second and third (and successive) lighter elements are instead required to meet the same criteria as the first: that the peak heights from these elements must match those found in the spectrum. But since these elements are light, it is likely that their signals will be superimposed on the signals produced by the heavier elements deeper in the sample. This situation is rectified by assuming some value for these heavy element "background" signals and then computing the values for the concentrations of the lighter elements in the first layer. When all concentrations have been computed in this layer, the values are adjusted proportionately to meet the constant stopping power criterion.

As the code continues to march deeper into the sample, it will eventually reach a layer in which the signal from the heaviest element will lie in the same channels as the signal produced by the lightest element in the first layer, resulting in a superposition of signals.
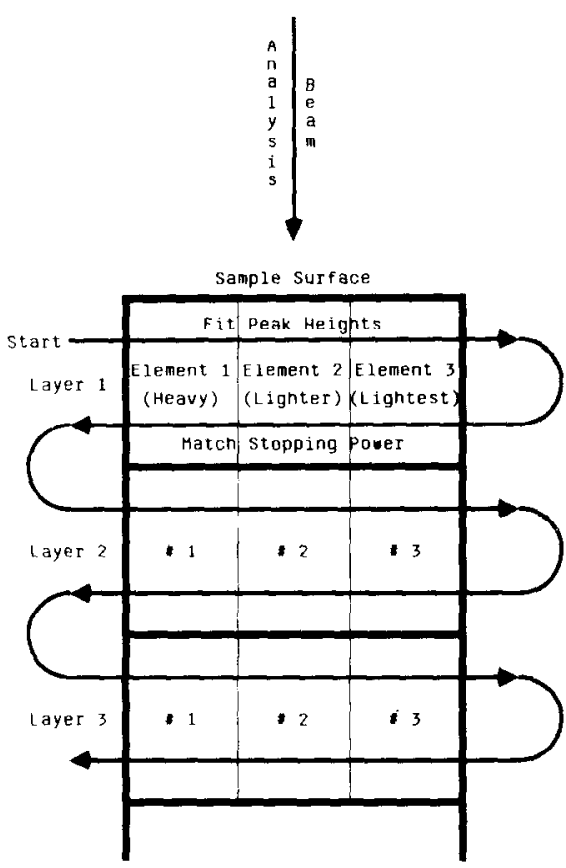

Fig. 2. Iteration structure of the code. The imner iterations are performed in each layer. One complete pass through all the layers counts as one outer iteration.
In performing the calculation for the heavy element concentration in this layer, it will almost certainly achieve a result different from the value assumed at the outset. This makes it necessary to repeat the calculation performed in the first layer. Therefore, the code must iterate through the layers, slightly shifting the concentrations in each layer on every pass. The corrections are made possible by the response matrices which detail the origin of each contribution to the total spectrum. The iteration proceeds from front to back in the samples, and from the heaviest to the lightest element in each layer, fig. 2. Convergence is tested after each complete pass through the sample by looking at the largest concentration change occurring for any element in any layer. In practice, the statistical fluctuations in real spectra will often induce small oscillations (one pair of adjacent layers exchanging compositions on successive iterations) which will cause the code to fail to detect convergence at fine tolerance levels. From experience with test cases, it has been found that little change is detected in most profiles after six complete iterations, and the code is typically. limited to ten iterations with a $1 \%$ convergence criterion. If convergence is not detected at this point, the profiles from successive iterations can be compared manually to check for significant variations.

\section{Verification on computer generated spectra}

Several test cases were run in which the code was used to fit self-generated computer spectra given an initially incorrect guess at the concentration profile. Not surprisingly, it performed quite well on these artificial problems, converging before the iteration limit in all cases with a $1 \%$ convergence criterion. One such test

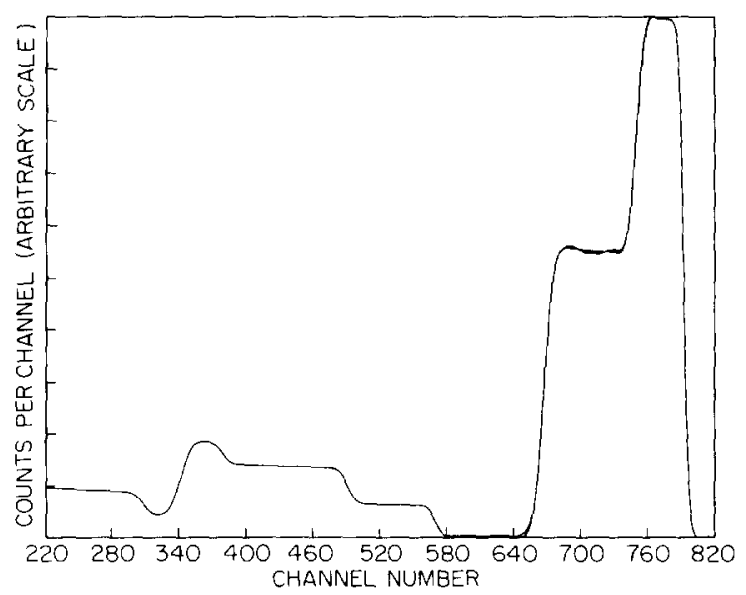

Fig. 3. Computer generated spectrum of a sample consisting of nickel deposited onto $\mathrm{SiO}_{2}$. 
case is given in fig. 3 , which shows the spectrum computed to result from a sample consisting of three layers: pure nickel, followed by a mixture of nickel, oxygen, and silicon, followed by a layer of silicon dioxide. The computer fit to this spectrum overlays the actual spectrum and the deviations are just barely visible. Two points of importance should be noted from these trials. First, very thin layers with low stopping powers $(15 \AA$ of aluminum) produce a very narrow signal with poor results and long convergence times. For example, while the average computed composition in four adjacent thin layers may be correct, the variance among all these layers will be quite large. Second, since the layer structure is determined by the initial guess, sharp interfaces may be spanned by a single layer resulting in an apparently blurred interface.

\section{Verification on experimentally generated spectra}

In order to verify the utility of the code, two and three element samples were prepared and RBS spectra were obtained for analysis. Four different samples were prepared, fig. 4. The nickel substrates were made from five nines purity nickel foil, vacuum annealed for five hours at $1100^{\circ} \mathrm{C}$, from which $3 \mathrm{~mm}$ disks were punched. These were successively mechanically polished, finishing with $0.05 \mu \mathrm{m}$ paste, and then electropolished using a jet thinner with a wide bore nozzle in a solution of $20 \%$ perchloric acid in ethanol. The $\mathrm{SiO}_{2}$ substrate was simply a clean microscope slide which showed no detectable fringe shifts when viewed under green light in an interferometer, thus assuring a surface smoothness of at
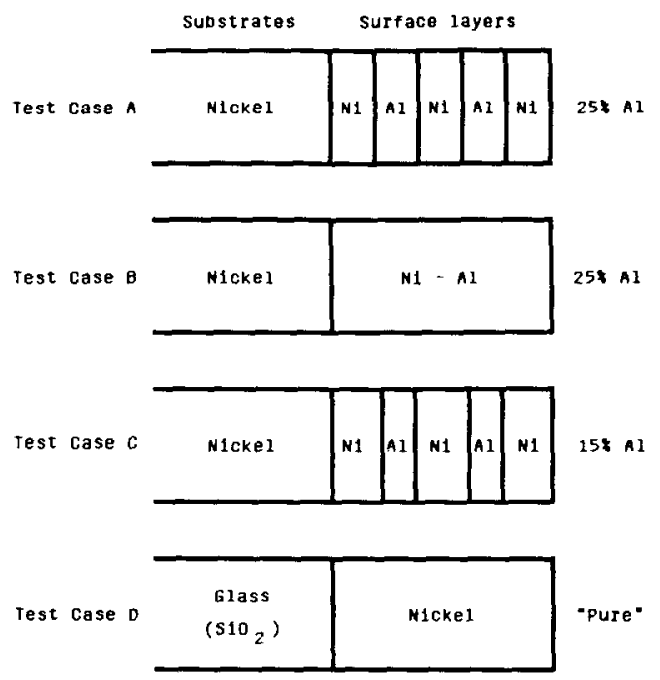

Fig. 4. Samples prepared for experimental verification of the code. least $500 \AA$. Samples were prepared in an oil-free vacuum evaporation chamber equipped with sorption and cryo pumps and a multiple hearth electron beam evaporation source. Layered samples were produced by switching between nickel and aluminum hearths. Layer thickness was controlled with an oscillating quartz crystal thickness monitor to achieve concentrations of 25 at. $\%$ and 15 at.\% aluminum in surfaces with a total thickness of approximately $600 \AA$. One 25 at.\% Al sample was vacuum annealed at $350^{\circ} \mathrm{C}$ for two hours to mix the surface layers. Subsequent TEM analysis of this sample revealed the presence of fcc superlattice rings in the diffraction pattern indicating the formation the intermetallic compound $\mathrm{Ni}_{3} \mathrm{Al}$. Typical evaporation parameters consisted of a pre-evaporation pressure of $10^{-7}$ Torr rising to $10^{-6}$ Torr during evaporation, and a deposition rate of $5 \AA / \mathrm{s}$.

Samples were mounted on an $X Y Z-\theta \phi$ manipulator stage and placed in the $15^{\circ}$ beamline target chamber at Argonne National Laboratory. The scattering and detection geometry is shown in fig. 5 and table 1 . Samples were tilted away from the detector in order to provide a high resolution geometry in which the surface layers would appear two to three times thicker due to the increased path length of particles traveling at oblique angles. Because of this tilting, it is important to know all the relevant angles quite precisely in order to avoid the large error associated with the uncertainty in the secant function at angles greater than $60^{\circ}$. This was accomplished by using the beamline laser to align samples in the path of the particle beam. Since our samples have mirror finishes, the reflected laser light can be used to precisely determine the relevant angles. Using this method, the detection and tilt angles were measured to within $0.1^{\circ}$.

The spectra were collected using a $1500 \mathrm{kV} \mathrm{He}-4$ beam of approximately $0.4 \mu \mathrm{A}$ passing through a $1 \mathrm{~mm}$ aperture. The collection system consists of a silicon surface barrier detector connected through a preamplifier to a linear amplifier equipped with pole-zero cancellation and active baseline restoration. The signals are analyzed with an analog to digital converter equipped

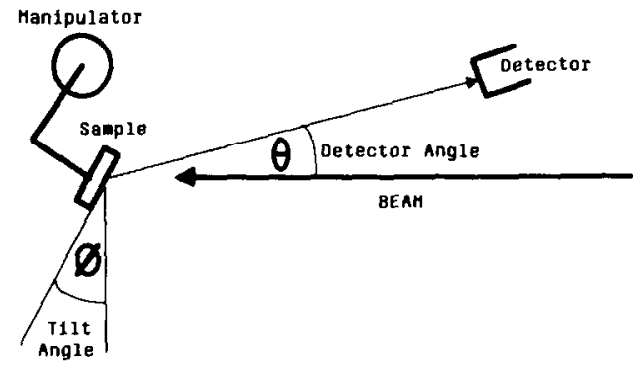

Fig. 5. Geometry of a backscattering experiment. 
Table 1

Values of detection angle $(\theta)$ and tilt angle ( $\phi$ ) for the four test cases.

\begin{tabular}{lll}
\hline $\begin{array}{l}\text { Test } \\
\text { sample }\end{array}$ & $\begin{array}{l}\theta \\
(\mathrm{deg})\end{array}$ & $\begin{array}{l}\phi \\
(\mathrm{deg})\end{array}$ \\
\hline A & 45.4 & 20.0 \\
B & 45.4 & 20.0 \\
C & 45.4 & 20.0 \\
D & 45.4 & 26.0
\end{tabular}

with pulse pile-up rejection provided by the amplifier and collected on a multichannel analyzer (MCA). Spectra were collected until 5000 counts accumulated in the leading edge channel. Collection times averaged about 20 min per spectrum. Standard spectra were collected from polished nickel and silicon blanks in order to provide an energy calibration for the MCA.
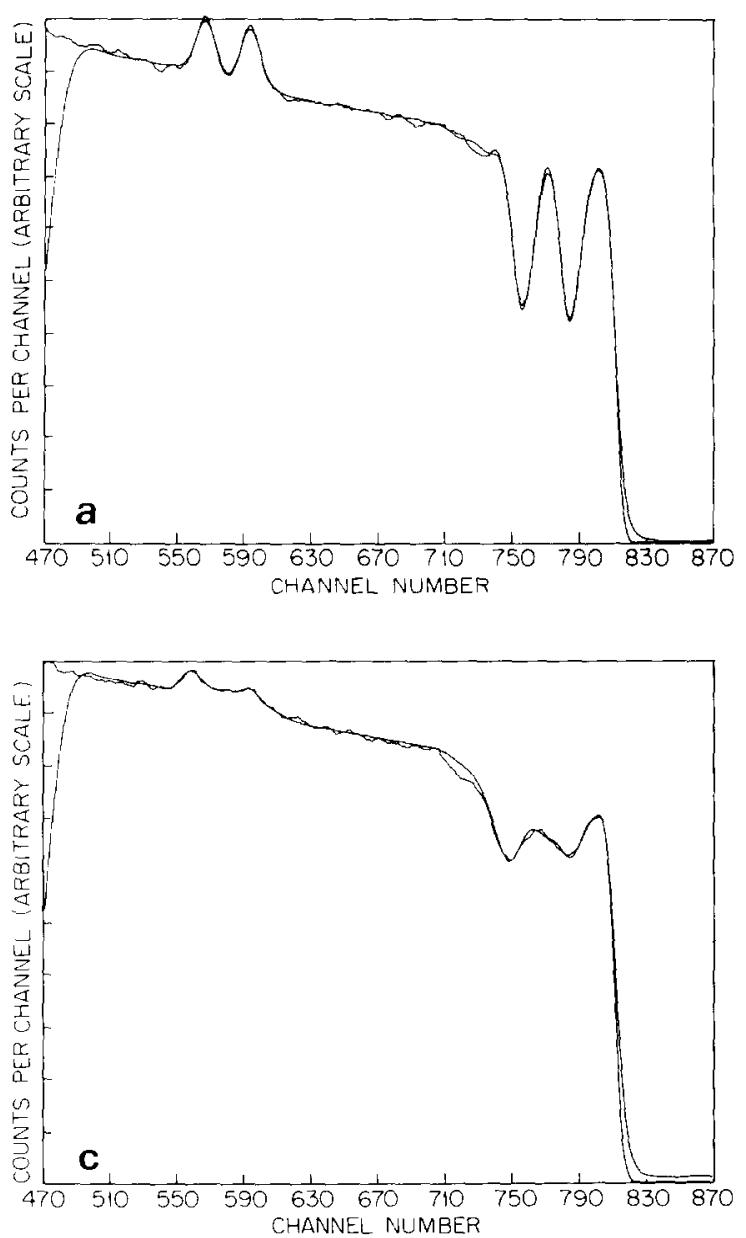

The raw data were smoothed with a 13-point least squares smoothing routine to eliminate statistical jitter. These spectra were fed as input to BASF along with the relevant geometry and beam energy information. Due to the lack of a good Faraday cup to measure total current, the three nickel-substrate spectra were normalized to a computed standard nickel spectrum, using the region between channels 620 and 690 where only the substrate signal is present. The $\mathrm{Ni}-\mathrm{SiO}_{2}$ spectrum lacks a good normalization region, so the nickel peak was used despite the fact that oxygen is present in the evaporated layer. The purpose of this sample is to test the ability of the code to distinguish a sharp interface in a multielement sample, so perfect normalization is not necessary.

\section{Results}

The smoothed spectra and the computed fits are shown in fig. 6 as a superposition of the smoothed and
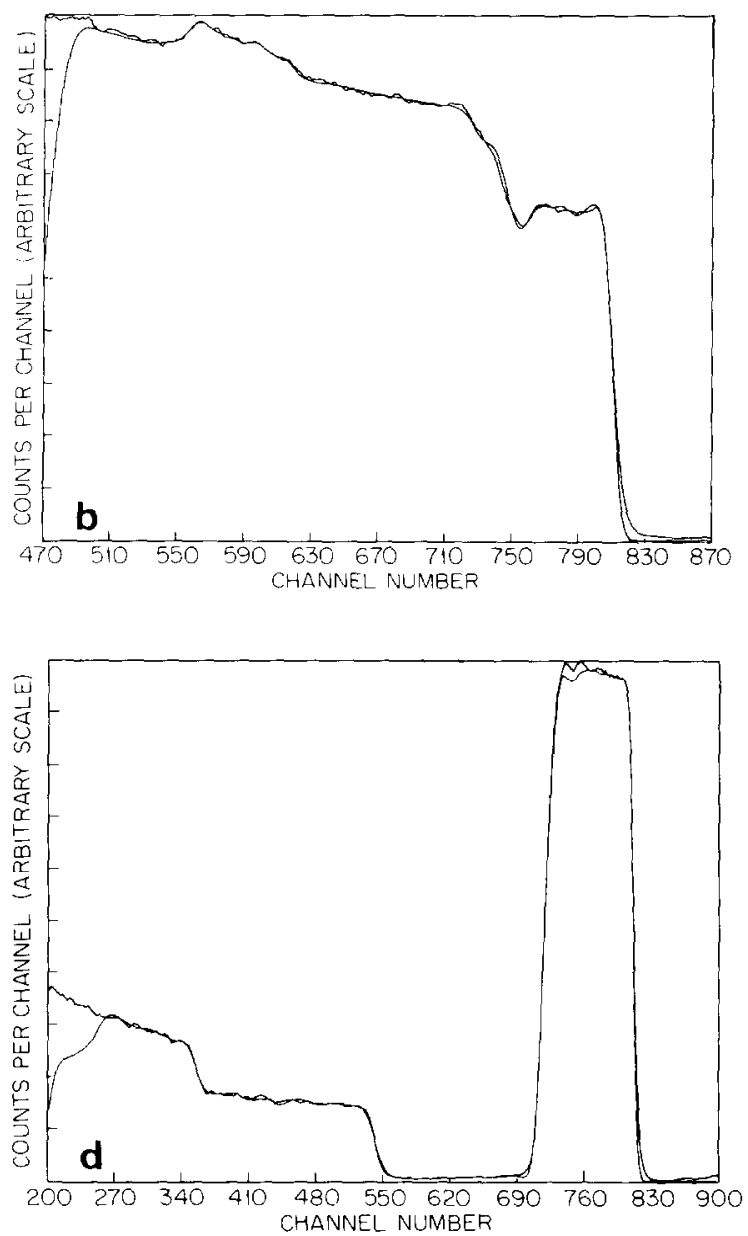

Fig. 6. Smoothed experimental spectra and computed fits for the four test cases. a) $\mathrm{Ni}-\mathrm{Al}$ layers, 25 at.\% $\mathrm{Al}, \mathrm{b}$ ) $\mathrm{Ni}-\mathrm{Al}$ thermally mixed, 25 at.\% Al, c) $\mathrm{Ni}-\mathrm{Al}$ layers, 15 at.\% $\mathrm{Al}$, and d) $\mathrm{Ni}$ on $\mathrm{SiO}_{2}$. 

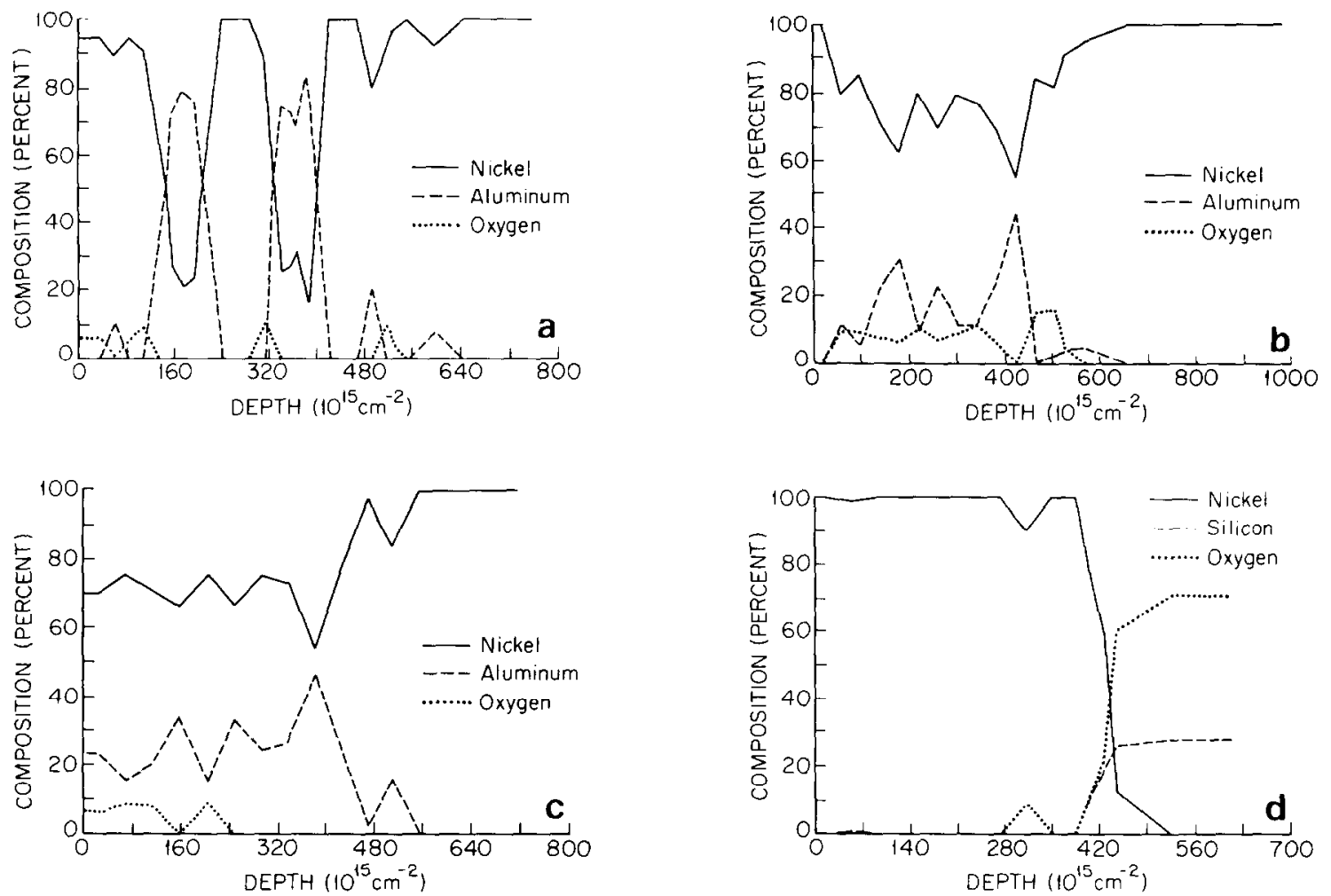

Fig. 7. Computed composition profiles for the test cases. a) $\mathrm{Ni}-\mathrm{Al}$ layers, 25 at.\% $\mathrm{Al}$, b) $\mathrm{Ni}-\mathrm{Al}$ thermally mixed. 25 at.\% $\mathrm{Al}$, c) $\mathrm{Ni}-\mathrm{Al}$ layers, 15 at.\% $\mathrm{Al}$, and d) $\mathrm{Ni}$ on $\mathrm{SiO}_{2}$.

fitted spectra. All the fits were achieved in ten iterations or less using the layer thicknesses recorded during the evaporation as an initial guess, except in case B, where an evenly mixed layer of $600 \AA$ of nickel and aluminum was assumed.

The ultimate value of this code is its ability to produce reasonably accurate concentration profiles. These are shown for the four test cases in fig. 7. Table 2 shows the tabular data from case A. Significantly, the total amount of aluminum calculated in cases $\mathrm{A}$ and $\mathrm{B}$ is nearly the same (within $3 \%$ ) although the impurity level in case B is nearly twice that in case A. Also, in case $\mathbf{B}$ it is possible to determine approximately the ratio of evaporated aluminum to nickel because the layers are so well mixed. The calculated ratio is within $1 \%$ of the thickness monitor readings taken during evaporation. The total amount of aluminum in the surface is within $5 \%$ of that measured by the thickness monitor. This holds for cases A, B, and C, and in all cases the code is consistently higher. The nickel-glass interface in case D shows up quite sharply over a $40 \AA$ layer. This demonstrates the ability of the code to pick out interfaces given well differentiated signals. In this case, however, the code cannot fit the nickel peak over its entire range, possibly due to the normalization problem mentioned earlier.

Inspection of the experimental spectra in cases $\mathrm{A}$ and $\mathrm{C}$ indicates the likely presence of an impurity in the

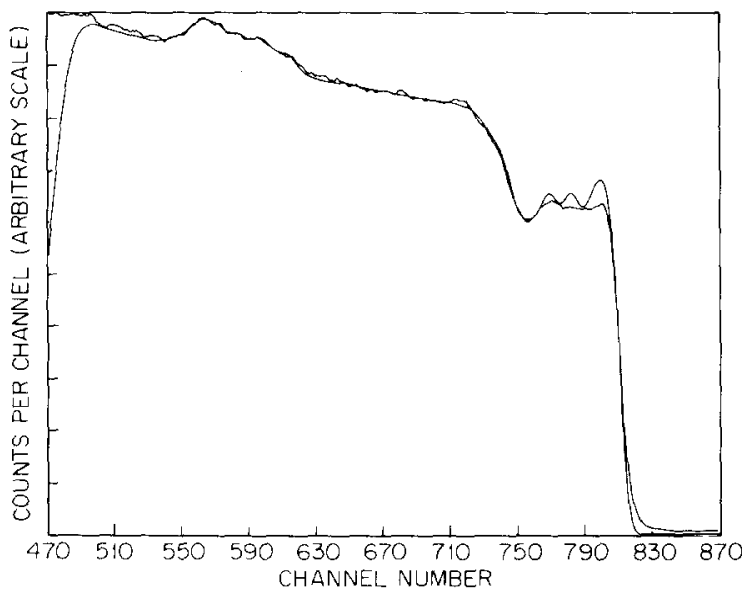

Fig. 8. Computed fit to test case B using only nickel and aluminum and no oxygen. 
evaporated nickel layers. This is evidenced by the lower peak heights in the evaporated layers as compared with the substrate. Since no peak arising from a heavy impurity is seen out beyond the nickel edge in the spectrum, a light impurity is assumed. A likely candidate for this impurity is oxygen, owing to the method of evaporation and the rise in pressure observed during the evaporation. Therefore, in cases A. B, and C the spectra were fit with nickel, aluminum, and oxygen. In case $\mathrm{D}$, silicon was substituted for aluminum.

The presence of an impurity in the surface is verified by the code when the spectrum from case B is analyzed alternately with and without oxygen. Fig. 8 shows the computed fit obtained when only nickel and aluminum are assumed to be present. Here it is apparent that the

Table 2

Computed composition profile from case $A$

\begin{tabular}{|c|c|c|c|c|}
\hline \multirow{2}{*}{$\begin{array}{l}\text { Layer } \\
\text { number }\end{array}$} & \multirow{2}{*}{$\begin{array}{l}\text { Thickness } \\
\text { (A) }\end{array}$} & \multicolumn{3}{|c|}{ Atom densities $\left(1 \times 10^{22} \mathrm{~cm}^{3}\right)$} \\
\hline & & Nickel & Aluminum & Oxygen \\
\hline 1 & 25.0 & 9.04 & 0.00 & 0.54 \\
\hline 2 & 25.0 & 9.02 & 0,00 & 0.57 \\
\hline 3 & 25.0 & 8.65 & 1.08 & 0.00 \\
\hline 4 & 25.0 & 9.04 & 0.00 & 0.54 \\
\hline 5 & 25.0 & 8.85 & 0.00 & 0.89 \\
\hline 6 & 25.0 & 6.91 & 3.79 & 0.00 \\
\hline 7 & 25.0 & 1.50 & 4.00 & 0.00 \\
\hline 8 & 25.0 & 1.19 & 4.49 & 0.00 \\
\hline 9 & 25.0 & 1.33 & 4.27 & 0.00 \\
\hline 10 & 25.0 & 6.68 & 4.14 & 0.00 \\
\hline 11 & 25.0 & 9.34 & 0.00 & 0.00 \\
\hline 12 & 25.0 & 9.34 & 0.00 & 0.00 \\
\hline 13 & 25.0 & 9.34 & 0.00 & 0.00 \\
\hline 14 & 25.0 & 8.77 & 0.00 & 1.02 \\
\hline 15 & 25.0 & 3.21 & 9.56 & 0.00 \\
\hline 16 & 25.0 & 1.71 & 3.68 & 0.00 \\
\hline 17 & 25.0 & 0.95 & 4.86 & 0.00 \\
\hline 18 & 25.0 & 2.49 & 2.47 & 0.00 \\
\hline 19 & 25.0 & 9.34 & 0.00 & 0.00 \\
\hline 20 & 25.0 & 9.34 & 0.00 & 0.00 \\
\hline 21 & 25.0 & 9.34 & 0.00 & 0.00 \\
\hline 22 & 25.0 & 8.03 & 2.04 & 0.00 \\
\hline 23 & 25.0 & 8.78 & 0.00 & 1.08 \\
\hline 24 & 50.0 & 9.13 & 0.00 & 0.00 \\
\hline
\end{tabular}

Note: Because RBS is sensitive only to depth measured in units of atoms $/ \mathrm{cm}^{2}$, the significant values in this table are actually the products of layer thickness and atom density.

\begin{tabular}{lll}
\hline $\begin{array}{l}\text { Comparisun of } \\
\text { computed and } \\
\text { measured values }\end{array}$ & \multicolumn{2}{l}{ Values in units of $1 \times 10^{15} \mathrm{at} . / \mathrm{cm}^{2}$} \\
\cline { 2 - 3 } (layers 1-23) & Computed & $\begin{array}{l}\text { Thickness } \\
\text { monitor value }\end{array}$ \\
\hline Total Ni & 387.7 & 379.8 \\
Total Al & 129.9 & 126.4 \\
Al fraction (\%) & 25.1 & 25.0 \\
\hline
\end{tabular}

code has found the proper amount of aluminum necessary to fit the spectrum in the region from channels 550 to 590 , but that the stopping power provided by this aluminum is not sufficient to reduce the nickel signal in the leading edge to the proper value. In fig. 6 , the inclusion of oxygen in the computations reveals that its presence at a level of approximately $5 \%$ in the surface will account for the reduced nickel peaks. Unfortunately, the oxygen signal is so far removed from the nickel signal and so much smaller compared to the nickel background, that it cannot be unambiguously determined that the impurity is indeed oxygen. All that can be said with certainty is that some light impurity such as oxygen or nitrogen or carbon is present to a small extent in the surface. This method of inferring the presence of an impurity without actually observing its backscattered signal was used in an analogous fashion by Borgesen [3] in the fitting program SQUEAKIE.

Although the code recognizes the nickel-glass interface in case $D$, cases $A$ and $C$ present more of a problem. In case $\mathrm{A}$ the interfaces are clearly present, but the aluminum concentration never rises to the full value of $100 \%$. In case $C$, even the presence of interfaces cannot be clearly seen. This could be due to three contributing factors: the aluminum layers may be too thin to be well resolved, or the system resolution may spread the signal too broadly, or else the layers are actually mixed at the interface. The latter could actually be the case due to the rapid diffusion which can occur in evaporated layers, but the lack of interface boundaries in case $C$ can only be due to a combination of the first two restrictions. Although aluminum layers in case $\mathrm{C}$ are only $65 \AA$ thick, the total amount of aluminum is accurately calculated in this case. All calculations resulted in convergence within 10 iterations which amounts to approximately $60 \mathrm{~s}$ on an Apollo DN320 computer.

\section{Discussion}

An iterative code has been developed which fits backscattering spectra obtained from multielement samples. This code has been tested on experimental data and has performed within the limits of the experiment. In particular, it has shown the capability to compute the total amount of aluminum in samples with different concentration profiles consistently, and it is at least as accurate as a thickness monitor for this application. Some comparisons between the code's results and the values measured by the thickness monitor are shown in table 3. The usefulness of the code is demonstrated in the verification of aluminum concentration in nickel-aluminum samples falling within the narrow two phase $\gamma / \gamma^{\prime}$ region of the nickel-aluminum phase diagram. Because the aluminum to nickel ratio is determinable to within $\pm 1 \%$, the equilibrium volume frac- 
tion of $\gamma^{\prime}$ in $\gamma$ can be controlled to within $\pm 10 \%$. This provides the much needed analysis capability for multilayer thin film preparation and thickness control. The code provides the ability to quickly analyze RBS spectra and could easily be interfaced with an MCA to provide on-line analysis, as is planned for the RBS facility being constructed at the University of Michigan.

Some drawbacks to this method of analysis should be noted. In order to obtain accurate results, the input parameters must be known precisely. Furthermore, this code shares the same limitations of all computer analysis codes, including the fact that it will not recognize or correct simple mistakes. For example, the code will not add new elements to the calculation in order produce a better fit. All isotopes present in the sample must be explicitly input at the heginning of the calculation. The code also assumes that an ideal RBS experiment is being analyzed. Therefore, the cross section used is always the classical Rutherford formula, multiple scattering is ignored, and the only allowance made for the non-point nature of the detector is the value of the detector energy resolution. All of these effects could prove significant in a given experiment, and the experimenter should be aware of the fact that the code will not automatically correct for them. Explicit code modifications could be made to account for these effects if they are found to be important in specific cases.

In the experimental verification of this code, some samples were prepared by evaporating nickel and aluminum layers onto unpolished beryllium foil substrates. The roughness and curvature of the surface resulted in spectra with very poorly defined nickel and aluminum peaks which spread into one another, fig. 9 . Upon analysis, the code managed to fit these spectra fairly well and even converged before the iteration limit. The resulting composition profiles, however, were in error by $20-30 \%$. This demonstrates the importance of providing smooth samples and good spectra for analysis.

There is room for improvement in some areas of the code. In particular, the greatest segment of computing time is allocated to spreading sharp signals over Gaussian distributions due to the modeling of the various

Table 3

Comparison of computed values with those measured using the thickness monitor during evaporation

\begin{tabular}{lccl}
\hline Sample & $\begin{array}{l}\text { Total aluminum } \\
\left(1 \times 10^{15} \mathrm{at} . / \mathrm{cm}^{2}\right)\end{array}$ & $\begin{array}{l}\text { Computed } \\
\text { aluminum } \\
\text { fraction } \\
(\%)\end{array}$ \\
\cline { 2 - 4 } & Monitor & Computed & \\
\hline A & 126.4 & 129.9 & 25.1 \\
B & 126.4 & 126.0 & 24.3 \\
C & 76.5 & 79.8 & 15.4 \\
\hline
\end{tabular}

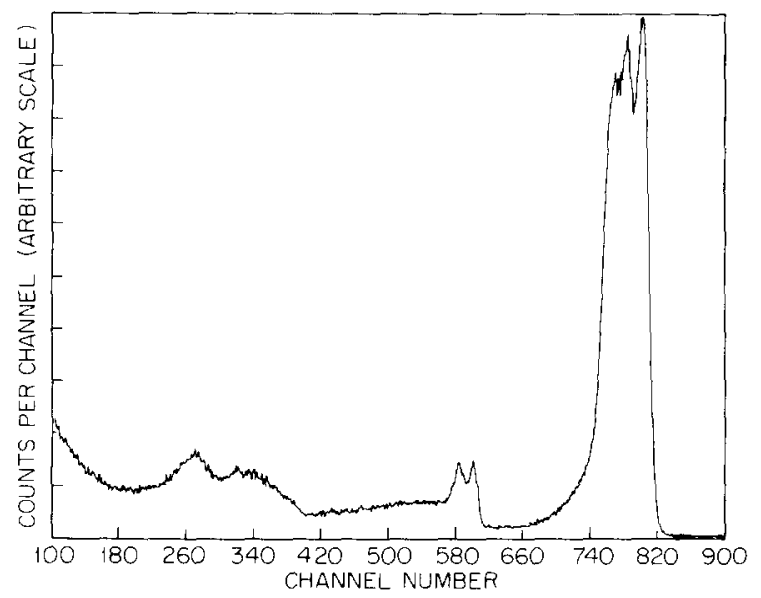

Fig. 9. RBS spectrum resulting from nickel and aluminum layers deposited on a rough beryllium foil surface.

spectrum broadening effects. If this process could be streamlined, perhaps by simply eliminating the error function evaluations, the running time could be cut in half. Also, some method of recognizing the mutually exclusive nature of some elements at certain depth regions, such as nickel in a glass slide, could help prevent needless iterations. These improvements, however, are of secondary importance since none of them should change the accuracy of the calculations.

One further limitation to any such attempt at RBS analysis is the accuracy of measured values of stopping powers. Ten percent variations in reported values are not uncommon [6]. The most recently measured values are used as a database, and these data generally show less scatter. In alloy samples, it is desirable to check the validity of Bragg's additivity rule with standards. In a well calibrated system with a good Faraday cup, this can be done by simply measuring the leading edge height in a spectrum obtained from an alloy of known composition. Unfortunately, the surface composition of alloys can frequently differ significantly from that of the bulk, resulting in errors. The accuracy of the stopping power values is one of the ultimate limitations on the resolving power of the techniquc. However, as it stands now, the code is quite useful in performing the analysis of evaporated thin layers of light elements on heavy substrates, which was the initial motivation for the work.

\section{Conclusion}

A computer code (BASF) has been constructed to perform automatic iterative fitting of Rutherford backscattering spectra using only the experimental spectrum and the parameter set defining the experiment. The 
code output consists of the total amount of each element present and a composition versus depth profile. The code can be used on samples containing anywhere from two to five elements.

On test cases involving evaporated layers of $\mathrm{Ni}$ (130 $\AA$ ) and $\mathrm{Al}(100 \AA$ ), the code was able to determine the element ratios to within $1 \%$ and total amounts to within $3 \%$ of thickness monitor readings taken during evaporation. The code was able to recognize slowly varying as well as sharply varying interfaces in layered samples of thickness on the order $100 \AA$. The code has a $1 \%$ convergence criterion and was able to solve all test cases within 10 iterations which amounts to less than $60 \mathrm{~s}$ on an Apollo DN320 computer. Limitations on the code and its uses include precise knowledge of the relevant experimental parameters used as input, specifications of all elements present in the sample, and an accurate knowledge of the computed stopping powers.
The authors would like to thank the Materials Science and Technology Division at Argonne National Laboratory for the use of their facilities in carrying out this work. In particular, the help of Lynn Rehn, Pete Baldo, Bernie Kestel, and Loren Thompson is greatly appreciated. This work was supported by NSF Grant DMR 8310032 .

\section{References}

[1] M. Schalit and R. Averback, unpublished (1980).

[2] L.R. Doolittle, Nucl. Instr, and Meth. B9 (1985) 344.

[3] P. Borgesen, R. Behrisch and B.M.U. Scherzer, Appl. Phys. A27 (1982) 183.

[4] Wei-Kan Chu, J. Mayer and M.-A. Nicolet, Backscattering Spectrometry (Academic Press, New York, 1981) p. 364

[5] J.E.E. Baglin and J.F. Ziegler, J. Appl. Phys. 45 (1974) 1413.

[6] H.H. Andersen and J.F. Ziegler, The Stopping Power and Range of lons in Matter, vols. 3 and 4 (Pergamon, New York, 1977). 